\title{
Stochastic Geometry Analysis and Additional Small Cell Deployment for HetNets Affected by Hot Spots
}

\author{
Nan E and Xiaoli Chu \\ Department of Electronic and Electrical Engineering, University of Sheffield, Sheffield S1 3JD, UK \\ Correspondence should be addressed to Xiaoli Chu; x.chu@sheffield.ac.uk
}

Received 7 September 2015; Revised 3 January 2016; Accepted 13 January 2016

Academic Editor: Pedro M. Ruiz

Copyright ( $) 2016$ N. E and X. Chu. This is an open access article distributed under the Creative Commons Attribution License, which permits unrestricted use, distribution, and reproduction in any medium, provided the original work is properly cited.

\begin{abstract}
Hot spots (HSs) of mobile users that were not expected in the original network planning may occur after a heterogeneous network (HetNet) has been deployed and affect the network performance. In this case, deploying additional small cells on top of the existing HetNet without changing the existing network infrastructure is considered as a solution. In this paper, we first provide a stochastic geometry analysis for a HetNet affected by a large HS and for the additional small cells that need to be deployed based on the spatial bivariate Poisson point process. The optimal numbers of additional small cells required in the HS and non-HS areas are obtained by minimizing the difference between the numbers of macrocell users after and before the HS occurs based on the analytical results. We then propose an algorithm to maximize the average user throughput by jointly optimizing the locations of additional small cells and user associations of all cells. Simulation results show that the proposed algorithm can maintain the average user throughput above a threshold with excellent fairness among all users even for a very high density of HS users.
\end{abstract}

\section{Introduction}

Small cell deployment in heterogeneous networks (HetNets) has been considered as an efficient solution to the rapid growth of mobile data demand under limited radio resources. It is anticipated that deploying low-power small cells will increase the area spectral efficiency [1]. In a HetNet, some small cells are deployed by the users, with their locations uncontrollable by the operators. Moreover, once a HetNet has been deployed, persistent hot spots (HSs) of user equipment (UE) that were not expected in the original network planning may occur, causing extra traffic demand. As the mobile traffic demand goes beyond the network capacity, the quality of service (QoS) of UE in the HetNet will be affected. In this case, deploying additional small cells on top of the existing HetNet by the operators would become necessary.

Considering cost effectiveness for network operators, it is desirable to optimize the number and locations of additional small cells to be deployed without changing the existing HetNet infrastructure. This relies on a thorough analysis of the HetNet. Stochastic geometry and the theory of random geometric graphs have been used in the analysis and design of wireless networks [2]. The internodal distances were modeled using a spatial bivariate Poisson point process (PPP) in [3]. However, the existence of significant HSs in HetNets has not been specifically considered in stochastic geometry based analysis of mobile networks.

In this paper, we analyse a HetNet before and after an unexpected HS occurs and additional small cells that would be required to mitigate the HS effect under a spatial bivariate PPP model. We model the spatial distributions of different network nodes (e.g., existing small cells, additional small cells to be deployed, HS UE, and non-HS UE) into five possible events. By minimizing the difference between the amounts of macrocell UE after and before the HS occurs based on the analytical results, the optimal numbers of additional small cells required for the HS and non-HS areas are obtained. We then propose an algorithm to maximize the average UE throughput in a HetNet affected by unexpected HS by jointly optimizing the locations of additional small cells on top of the existing HetNet and the user associations of all cells. The relationship between HS UE intensity and additional small cell intensities in HS and non-HS areas is analysed. The simulation results show that our proposed algorithm can effectively maintain the average UE throughput requirement with 
excellent fairness among all UE even for a very high intensity of HS UE.

The rest of the paper is organized as follows. In Section 2, the system model is presented. The analysis based on the spatial bivariate PPP and the analytical results are provided in Section 3. The optimization of additional small cell deployment and the algorithm to solve it are provided in Section 4. Simulation results and performance evaluation are presented in Section 5. Conclusions are drawn in Section 6.

\section{Related Work}

Using the stochastic geometry analysis, the authors of [3] studied the distribution of the distances between an arbitrary point and its dissimilar neighboring points as a spatial bivariate Poisson point process. In this paper we will extend the model of [3] into five events for our stochastic geometry analysis in Section 3.

As for small cell deployment in HetNets, the femtocell deployment with an arbitrary topology was optimized by maximizing the number of users supported with QoS constraints in [4]. In [5], the deployment locations of macro base stations (BSs), pico-BSs, and relays are optimized by selecting a subset of candidate sites. In [6], the locations of small cells are sequentially determined by maximizing the weighted sum of the received signal strength and load, but without considering the user associations. The small cell deployment strategy in [7] considering the movement of UE is proposed.

Although HS mitigation has been studied for wireless sensor networks [8], most existing works on small cell deployment strategies have focused on optimizing the deployment of small cells on top of a conventional macrocell network or the deployment of a whole new HetNet from scratch [4-6]. In [9], the dynamic placement of small cells for hot spots is optimized by minimizing the data delivery cost and by minimizing macrocell utilization, respectively, where the locations of additional small cells are chosen from the set of known candidate sites. As a result, the maximum number of additional small cells is limited by the number of candidate locations.

\section{System Model}

We consider a two-tier HetNet that contains macrocells and small cells. In each macrocell coverage area, the location of the macro-BS is constant, while the small cell BSs and UE are considered as random points [10]. Based on the spatial bivariate PPP [3], we define five events for the problem of deploying additional small cells on top of an existing HetNet as presented in Table 1. Event A represents non-HS UE (which is not jointly distributed with event $\mathrm{C}$, which will be explained later), with the spatial intensity $\alpha$. Event B denotes the HS UE (which is not jointly distributed with event $\mathrm{E}$, which will be explained later) in the HS area with the spacial intensity $\beta$. Since the HS area overlaps with the macrocell coverage, nonHS UE may also occur in the HS area. Event $\mathrm{C}$ represents the existing small cells. We assume that each existing small cell serves at least one non-HS UE and that each existing small cell BS is jointly distributed with a non-HS UE. Hence, event $\mathrm{C}$ is considered as an existing small cell and a non-HS
TABLE 1: Events.

\begin{tabular}{llcc}
\hline Event & Type & Intensity & Given \\
\hline A & Non-HS UE & $\alpha$ & Yes \\
B & HS UE & $\beta$ & Yes \\
C & Existing small cells & $\nu$ & Yes \\
D & Additional small cells & $\mu$ & No \\
& outside HS & & \\
E & Additional small cells in & $\rho$ & No \\
\hline
\end{tabular}

UE jointly occurring at a random point $y$. Event $\mathrm{D}$ denotes additional small cells that will be deployed in non-HS areas, with intensity $\mu$. Event $\mathrm{E}$ denotes additional small cells deployed in the HS area with intensity $\rho$, each serving at least one HS UE. Thus, each event E node is jointly distributed with a HS UE.

After a significant HS occurs and additional small cells are deployed, the overall spatial intensity of small cells in non-HS areas is $\nu+\mu$ and that of small cells in the HS area is $\nu+\rho$ [11].

For event $\mathrm{C}$, denote $f_{1}\left(\left\|x_{\mathrm{U}}-y\right\|\right)$ and $g_{1}\left(\left\|x_{\mathrm{S}}-y\right\|\right)$ as the probability density functions (PDFs) of a UE at $x_{U}$ and an existing small cell at $x_{\mathrm{S}}$ when event $\mathrm{C}$ occurs at $y$, respectively. Zero-mean isotopic Gaussian distributions with standard deviations $\sigma_{f_{1}}$ and $\sigma_{g_{1}}$ are chosen for $f_{1}(X)$ and $g_{1}(X)$, respectively [3].

Before the HS occurs, the probability of event C occurring at any point in the macrocell coverage area is calculated as [3]

$$
\begin{aligned}
\operatorname{Pr}\{\mathrm{C}\} & =h_{1}\left(x_{\mathrm{U}}, x_{\mathrm{S}}\right) \\
& \triangleq \int_{y \in \mathscr{R}_{\mathrm{M}}} f_{1}\left(\left\|x_{\mathrm{U}}-y\right\|\right) g_{1}\left(\left\|x_{\mathrm{S}}-y\right\|\right) d y \\
& =h_{1}\left(x_{\mathrm{U}}-x_{\mathrm{S}}, 0\right)=h_{1}\left(X_{1}, 0\right) \\
& =\frac{1}{2 \pi \sigma_{1}^{2}} e^{-\left\|X_{1}\right\|^{2} / 2 \sigma_{1}^{2}},
\end{aligned}
$$

where $\mathscr{R}_{\mathrm{M}}$ is the macrocell coverage area, $X_{1}=x_{\mathrm{U}}-x_{\mathrm{S}}$, and $\sigma_{1}^{2}=\sigma_{f_{1}}^{2}+\sigma_{g_{1}}^{2}$, which is a correlation factor that is determined by the distance of a non-HS UE to its jointly distributed existing small cell BS for event C.

Similarly, for event $\mathrm{D}$, denote $f_{2}\left(\left\|x_{\mathrm{HSU}}-y\right\|\right)$ and $g_{2}\left(\| x_{\mathrm{NS}}-\right.$ $y \|)$ as the PDFs for a HS UE occurring at $x_{\mathrm{HSU}}$ and an additional small cell BS deployed at $x_{\mathrm{NS}}$ in the HS area, respectively. $f_{2}(X)$ and $g_{2}(X)$ are zero-mean isotopic Gaussian distributions with standard deviations $\sigma_{f_{2}}$ and $\sigma_{g_{2}}$, respectively. The probability of a joint event between a HS UE at $x_{\mathrm{HSU}}$ and an additional small cell BS at $x_{\mathrm{NS}}$ occurring in the HS area is given by [3]

$$
\begin{aligned}
h_{2} & \left(x_{\mathrm{HSU}}, x_{\mathrm{NS}}\right) \\
& \triangleq \int_{y \in \mathscr{R}_{\mathrm{H}}} f_{2}\left(\left\|x_{\mathrm{HSU}}-y\right\|\right) g_{2}\left(\left\|x_{\mathrm{NS}}-y\right\|\right) d y \\
& =h_{2}\left(X_{2}, 0\right)=\frac{1}{2 \pi \sigma_{2}^{2}} e^{-\left\|X_{2}\right\|^{2} / 2 \sigma_{2}^{2}},
\end{aligned}
$$

where $\mathscr{R}_{\mathrm{H}}$ is the HS area, $X_{2}=x_{\mathrm{HSU}}-x_{\mathrm{NS}}$, and $\sigma_{2}^{2}=\sigma_{f_{2}}^{2}+\sigma_{g_{2}}^{2}$. 
3.1. The Network without HS. By using the given intensities of event $\mathrm{A}$ and event $\mathrm{C}$ and $h_{1}\left(x_{\mathrm{U}}, x_{\mathrm{S}}\right)$ in (1), we can calculate the expected amount of non-HS UE in the coverage of an existing small cell BS at $x_{\mathrm{S}}, x_{\mathrm{S}} \in \mathscr{R}_{\mathrm{M}}$, as follows:

$$
\begin{aligned}
\chi_{\mathrm{AC}}\left(r_{\mathrm{S}}\right) & =\int_{x_{\mathrm{U}} \in \mathscr{C}_{\mathrm{S}}}\left(\alpha+h_{1}\left(x_{\mathrm{U}}, x_{\mathrm{S}}\right)\right) d x_{\mathrm{U}} \\
& =\alpha \pi r_{\mathrm{S}}^{2}+\left(1-e^{-r_{\mathrm{S}}^{2} / 2 \sigma_{1}^{2}}\right),
\end{aligned}
$$

where $\mathscr{C}_{\mathrm{S}}$ is the coverage area of a small cell and $r_{\mathrm{S}}$ is the coverage radius of a small cell.

The expected number of existing small cells in the macrocell coverage area is given by

$$
\chi_{\mathrm{C}}\left(r_{\mathrm{M}}\right)=\int_{x_{\mathrm{S}} \in \mathscr{R}_{\mathrm{M}}} v d x_{\mathrm{S}}=v \pi r_{\mathrm{M}}^{2}
$$

where $r_{M}$ is macrocell coverage radius.

The expected total amount of non-HS UE in the macrocell coverage area before HS occurs is given by

$$
\begin{aligned}
E\left\{N_{\mathrm{U}}\right\} & =\int_{x_{\mathrm{U}} \in \mathscr{R}_{\mathrm{M}}} \alpha d x_{\mathrm{U}}+\int_{x_{\mathrm{U}} \in \mathscr{R}_{\mathrm{M}}} v h_{1}\left(x_{\mathrm{U}}, x_{\mathrm{S}}\right) d x_{\mathrm{U}} \\
& =\alpha \pi r_{\mathrm{M}}^{2}+\left(1-e^{-r_{\mathrm{M}}^{2} / 2 \sigma_{1}^{2}}\right) \nu \pi r_{\mathrm{M}}^{2}
\end{aligned}
$$

3.2. The Network with HS. After a HS with UE intensity $\beta$ occurs in the coverage of a macrocell, by using $h_{2}\left(x_{\mathrm{HSU}}, x_{\mathrm{NS}}\right)$ in (2), the expected amount of UE (including HS UE and nonHS UE) in an additional small cell coverage which is in the HS area can be written as

$$
\begin{aligned}
\chi_{\mathrm{ABE}}\left(r_{\mathrm{S}}\right)= & \int_{x_{\mathrm{HSU}} \in \mathscr{C}_{\mathrm{S}}}\left(\beta+h_{2}\left(x_{\mathrm{HSU}}, x_{\mathrm{NS}}\right)\right) d x_{\mathrm{HSU}} \\
& +\int_{x_{\mathrm{U}} \in \mathscr{C}_{\mathrm{S}}} \alpha d x_{\mathrm{U}} \\
= & (\alpha+\beta) \pi r_{\mathrm{S}}^{2}+\left(1-e^{-r_{\mathrm{S}}^{2} / 2 \sigma_{2}^{2}}\right),
\end{aligned}
$$

where $\sigma_{2}$ is defined in (2).

The expected amount of UE (including HS UE and nonHS UE) in an existing small cell coverage that overlaps with the HS area is given by

$$
\begin{aligned}
\chi_{\mathrm{AB}}\left(r_{\mathrm{S}}\right) & =\chi_{\mathrm{AC}}\left(r_{\mathrm{S}}\right)+\int_{x_{\mathrm{HSU}} \in \mathscr{C}_{\mathrm{S}}} \beta d x_{\mathrm{HSU}} \\
& =(\alpha+\beta) \pi r_{\mathrm{S}}^{2}+\left(1-e^{-r_{\mathrm{S}}^{2} / 2 \sigma_{1}^{2}}\right),
\end{aligned}
$$

where $\mathscr{C}_{\mathrm{h}}$ is the area of the small cell coverage in the HS area. For simplicity, we assume that the existing small cell is completely in the HS area in (7).

The expected amount of non-HS UE served by additional small cells in non-HS areas is given by

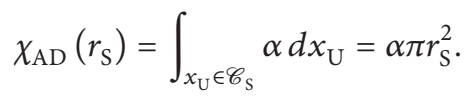

The expected number of existing small cells in non-HS areas is

$$
\chi_{\mathrm{C}}^{\prime}=\int_{x_{\mathrm{S}} \in \mathscr{R}_{\mathrm{nH}}} v d x_{\mathrm{S}}=v \pi\left(r_{\mathrm{M}}^{2}-r_{\mathrm{HS}}^{2}\right) \text {, }
$$

where $\mathscr{R}_{\mathrm{nH}}$ is the non-HS area in the macrocell coverage.

The expected number of additional small cells in the HS area is given by

$$
\chi_{\mathrm{E}}\left(r_{\mathrm{HS}}\right)=\int_{x_{\mathrm{NS}} \in \mathscr{R}_{\mathrm{H}^{\prime}}} v d x_{\mathrm{NS}}=\rho\left(\pi r_{\mathrm{HS}}^{2}-v \pi^{2} r_{\mathrm{HS}}^{2} r_{\mathrm{S}}^{2}\right),
$$

where $r_{\mathrm{HS}}$ is the radius of the HS area, which is assumed to be a disc area, and $\mathscr{R}_{\mathrm{H}^{\prime}}$ is the HS area excluding the coverage area of existing small cells in the HS. Since the HS area overlaps with the existing network, the coverage areas of existing small cells located in the HS need to be removed from the calculation of additional small cells in the HS area.

The expected number of existing small cells in the HS area is given by $\chi_{\mathrm{C}}\left(r_{\mathrm{HS}}\right)$ following (5).

The expected number of additional small cells in non-HS areas is

$$
\chi_{\mathrm{D}}=\int_{x_{\mathrm{NS}} \in \mathscr{R}_{\mathrm{nH}}} \mu d x_{\mathrm{NS}}=\mu \pi\left(r_{\mathrm{M}}^{2}-r_{\mathrm{HS}}^{2}\right) .
$$

The expected total amount of UE in the macrocell coverage area after the HS occurs is given by

$$
\begin{aligned}
E\left\{N_{\mathrm{U}}^{\prime}\right\}= & \alpha \pi r_{\mathrm{M}}^{2}+\nu \pi r_{\mathrm{M}}^{2}\left(1-e^{-r_{\mathrm{M}}^{2} / 2 \sigma_{1}^{2}}\right)+\beta \pi r_{\mathrm{HS}}^{2} \\
& +\rho\left(\pi r_{\mathrm{HS}}^{2}-v \pi^{2} r_{\mathrm{HS}}^{2} r_{\mathrm{S}}^{2}\right)\left(1-e^{-r_{\mathrm{HS}}^{2} / 2 \sigma_{2}^{2}}\right) .
\end{aligned}
$$

\section{Intensity of Additional Small Cells}

We assume that all the UE in the HetNet is satisfied with its service before a significant HS occurs. After a significant HS occurs, without deploying additional small cells, the HS UE that cannot be accommodated by existing small cells will be served by the macrocell. This extra burden on the macro-BS may reduce the QoS of macrocell UE. In order to avoid overloading the macrocell, we aim to keep the amount of macrocell UE after the HS occurs as close as possible to that before the HS occurs.

The expected amount of macrocell UE before the HS occurs is given by

$$
E\left\{N_{\mathrm{MU}}\right\}=E\left\{N_{\mathrm{U}}\right\}-\chi_{\mathrm{AC}}\left(r_{\mathrm{S}}\right) \cdot \chi_{\mathrm{C}}\left(r_{\mathrm{M}}\right) .
$$

After the HS occurs and additional small cells are deployed, the expected amount of UE served by the existing small cells in non-HS areas can be calculated as

$$
E\left\{N_{\mathrm{NHS}}\right\}=\chi_{\mathrm{AC}}\left(r_{\mathrm{S}}\right) \cdot \chi_{\mathrm{C}}^{\prime}
$$

The expected amount of UE served by the existing small cells in the HS area can be calculated as

$$
E\left\{N_{\mathrm{HS}}^{\prime}\right\}=\chi_{\mathrm{AB}}\left(r_{\mathrm{S}}\right) \cdot \chi_{\mathrm{C}}\left(r_{\mathrm{HS}}\right) .
$$


The expected amount of UE served by additional small cells in the HS area can be calculated as

$$
E\left\{N_{\mathrm{HS}}\right\}=\chi_{\mathrm{ABE}}\left(r_{\mathrm{S}}\right) \cdot \chi_{\mathrm{E}}\left(r_{\mathrm{HS}}\right) .
$$

The expected amount of UE served by additional small cells in non-HS areas can be calculated as

$$
E\left\{N_{\mathrm{NHS}}^{\prime}\right\}=\chi_{\mathrm{AD}}\left(r_{\mathrm{S}}\right) \cdot \chi_{\mathrm{D}}
$$

Hence, the expected amount of macrocell UE after the HS occurs can be calculated as

$$
\begin{aligned}
E\left\{N_{\mathrm{MU}}^{\prime}\right\} & \\
= & E\left\{N_{\mathrm{U}}^{\prime}\right\} \\
& -\left(E\left\{N_{\mathrm{NHS}}\right\}+E\left\{N_{\mathrm{HS}}\right\}+E\left\{N_{\mathrm{HS}}^{\prime}\right\}+E\left\{N_{\mathrm{NHS}}^{\prime}\right\}\right) .
\end{aligned}
$$

We optimize the intensities of additional small cells in the HS and non-HS areas through minimizing the difference between the amounts of macrocell UE after and before the HS occurs as follows:

$$
\begin{aligned}
\underset{\rho, \mu}{\arg \min } & \left|E\left\{N_{\mathrm{MU}}^{\prime}\right\}-E\left\{N_{\mathrm{MU}}\right\}\right| \\
\text { s.t. } & \rho, \mu \geq 0 .
\end{aligned}
$$

By using the results in (3)-(18), the objective function in (19) can be rewritten as

$$
\begin{aligned}
& \underset{\rho, \mu}{\arg \min }|a \cdot \rho+b \cdot \mu+c|, \\
& a= {\left[(\alpha+\beta) \pi r_{\mathrm{S}}^{2}+\left(1-e^{-r_{\mathrm{S}}^{2} / 2 \sigma_{2}^{2}}\right)\right] } \\
& \cdot\left(\pi r_{\mathrm{HS}}^{2}-v \pi^{2} r_{\mathrm{HS}}^{2} r_{\mathrm{S}}^{2}\right), \\
& b= \alpha \pi r_{\mathrm{S}}^{2}\left(\pi r_{\mathrm{M}}^{2}-\pi r_{\mathrm{HS}}^{2}\right), \\
& c=\left(\beta \pi r_{\mathrm{S}}^{2}+e^{-r_{\mathrm{S}}^{2} / 2 \sigma_{1}^{2}}-e^{-r_{\mathrm{S}}^{2} / 2 \sigma_{2}^{2}}\right) \nu \pi r_{\mathrm{HS}}^{2}-\beta \pi r_{\mathrm{HS}}^{2} .
\end{aligned}
$$

The optimization problem in (19) can be readily solved using numerical methods. Here we present numerical solutions of the optimal $\rho$ and $\mu$ obtained under the system parameters in Table 2 . The center of the HS area is randomly generated in the macrocell coverage area while ensuring the whole HS area is within the macrocell coverage area with a minimum distance from the macrocell BS of $100 \mathrm{~m}$. The intensity of additional small cells in the HS area versus the intensity of HS UE is presented in Figure 1. It can be seen that the intensity of additional small cells in the HS area increases with the intensity of HS UE at a decreasing rate. Figure 2 shows the intensity of additional small cells in non-HS areas versus the intensity of HS UE. Comparing Figure 2 with Figure 1, the intensity of additional small cells in the HS area is much higher than that in non-HS areas; the rapid increase of the intensity for additional small cells in non-HS areas when $\beta<1000 \mathrm{UE} / \mathrm{km}^{2}$ is because the intensity of additional small cells in the HS area is not enough to serve all the HS UE,
TABLE 2: System setting.

\begin{tabular}{lc}
\hline Parameter & Value \\
\hline$\alpha$ & $50 \mathrm{UE} / \mathrm{km}^{2}$ \\
$\nu$ & $10 \mathrm{BS} / \mathrm{km}^{2}$ \\
$\sigma_{1}^{2}$ & 0.1 \\
$\sigma_{2}^{2}$ & 0.1 \\
$r_{\mathrm{M}}$ & $500 \mathrm{~m}$ \\
$r_{\mathrm{S}}$ & $20 \mathrm{~m}$ \\
$r_{\mathrm{HS}}$ & $50 \mathrm{~m}$ \\
\hline
\end{tabular}

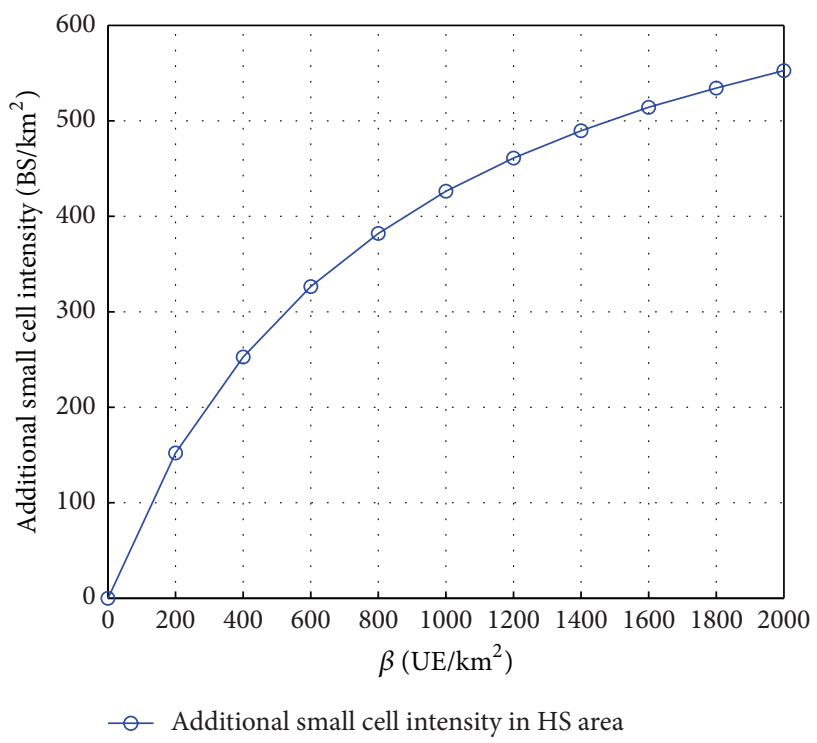

FIGURE 1: The intensity of additional small cells in the HS area $(\rho)$ versus the intensity of HS UE.

thus requiring additional small cells in non-HS area to share the extra burden caused by HS UE to the cellular network. For large values of $\beta$, the intensity of additional small cells in non-HS areas decreases with $\beta$, because the additional small cells in the HS area are now able to serve the majority of HS UE. Figure 3 shows the expected numbers of additional small cells in the HS and non-HS areas under the setting in Table 2.

\section{Deployment of Additional Small Cells}

Based on the analytical results in Section 3, we propose to maximize the average UE throughput among all UE by jointly optimizing the locations of new small cells and user associations of all cells for a HetNet affected by a HS not expected in the original network planning.

We consider the downlink (DL) of a two-tier HetNet consisting of one central macrocell and $\left\lceil\chi_{\mathrm{C}}\left(r_{\mathrm{M}}\right)\right\rceil$ small cells randomly distributed in the macrocell coverage area. The number of existing BSs is $N_{\mathrm{eBS}}=1+\left\lceil\chi_{\mathrm{C}}\left(r_{\mathrm{M}}\right)\right\rceil$. Each cell has access to the total of $N_{\mathrm{RB}}$ resource blocks (RBs). Denoting the number of additional small cells to be deployed in the HS area as $N_{\mathrm{NS}_{1}}$ and the new small cells to be deployed in non-HS area as $N_{\mathrm{NS}_{2}}$, the total number of BSs is given by $N_{\mathrm{BS}}=N_{\mathrm{eBS}}+$ $N_{\mathrm{NS}_{1}}+N_{\mathrm{NS}_{2}}$. Denote the set of BSs as $\mathcal{N}_{\mathrm{BS}}=\left\{1,2, \ldots, N_{\mathrm{BS}}\right\}$. 


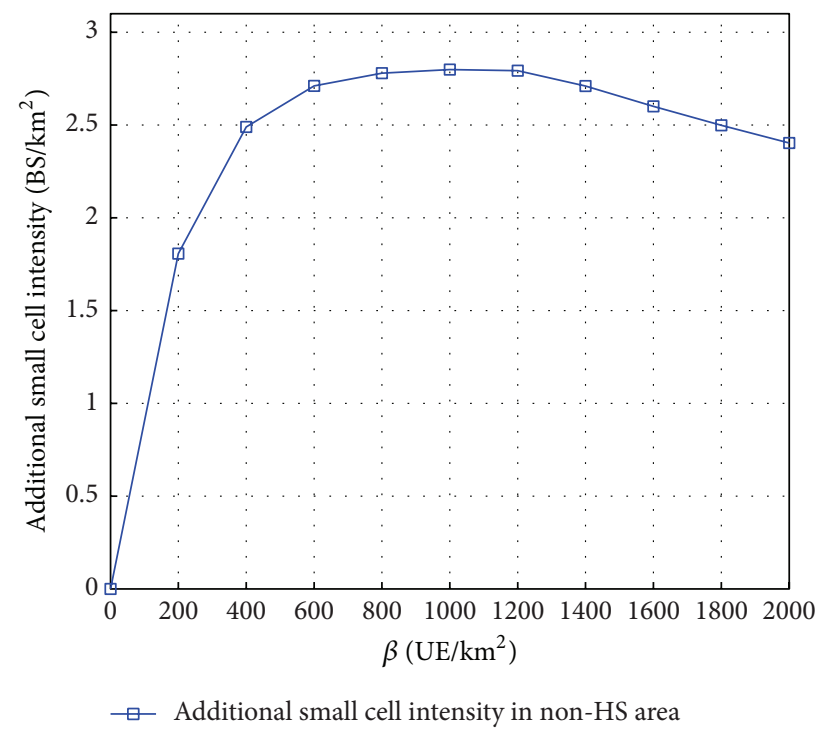

FIGURE 2: The intensity of additional small cells in non-HS areas $(\mu)$ versus the intensity of HS UE.

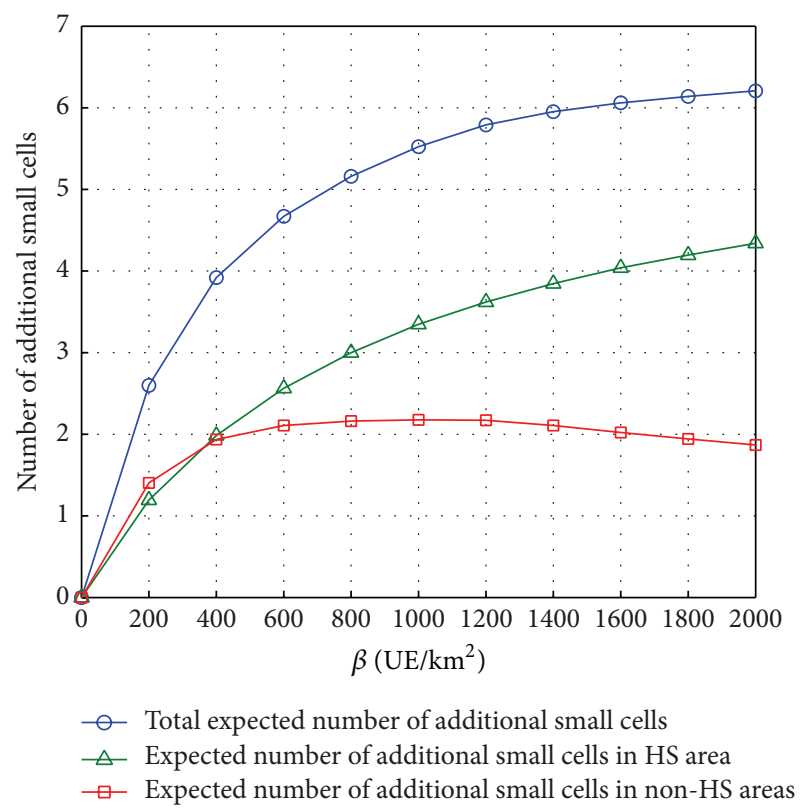

Figure 3: An example of the total number of new small cells under the setting in Table 2 .

According to the system model in Section 2, the expected amount of HS UE is given by

$$
\chi_{\mathrm{BE}}\left(r_{\mathrm{HS}}\right)=\beta \pi r_{\mathrm{HS}}^{2}+\left(1-e^{-r_{\mathrm{HS}}^{2} / 2 \sigma_{2}^{2}}\right) v \pi r_{\mathrm{HS}}^{2} .
$$

Let $N_{\mathrm{NHS}}=\left\lceil E\left\{N_{\mathrm{U}}\right\}\right\rceil$ denote the amount of non-HS UE and let $N_{\mathrm{HS}}=\left\lceil\chi_{\mathrm{BE}}\left(r_{\mathrm{HS}}\right)\right\rceil$ denote the amount of HS UE in the macrocell coverage area. The set of all UE is given by $\mathcal{N}_{\mathrm{U}}=$ $\left\{1,2, \ldots, N_{\mathrm{U}}\right\}$, where $N_{\mathrm{U}}=N_{\mathrm{NHS}}+N_{\mathrm{HS}}$. by

The throughput of the $i$ th $\mathrm{UE}\left(i=\left\{1,2, \ldots, N_{\mathrm{U}}\right\}\right)$ is given

$$
\gamma_{i}=\sum_{j=1}^{N_{\mathrm{BS}}} N_{j}^{\mathrm{RB}} \cdot W \cdot \log _{2}\left(1+\frac{P_{j} \cdot g_{i, j} \cdot u_{i, j}}{I_{i, j}+N_{0}}\right)
$$

where $u_{i, j}=1$ if the $i$ th UE is served by the $j$ th BS and $u_{i, j}=0$ otherwise; $W$ is the bandwidth of a RB; without considering power control, $P_{j}$ is the DL transmit power of the $j$ th $\mathrm{BS}$ in a RB; and $P_{j}=P_{\mathrm{M}(S)}$ if it is a macro-BS (small-cell BS). Assuming that the channel on each RB sees independent and identical Rayleigh fading, the channel power gain of the link between the $i$ th $\mathrm{UE}$ and the $j$ th $\mathrm{BS}$ in a RB is expressed as

$$
g_{i, j}=g_{\mathrm{f}, i j} \cdot g_{\mathrm{pl}, i j},
$$

where $g_{\mathrm{f}, i j}$ is the exponentially distributed fading gain with unit mean and $g_{\mathrm{pl}, i j}$ is the path loss as given in [1]; $N_{j}^{\mathrm{RB}}$ is the number of RBs per UE in cell $j$ and is given by

$$
N_{j}^{\mathrm{RB}}=\left\lfloor\frac{N_{\mathrm{RB}}}{\sum_{i=1}^{N_{\mathrm{U}}} u_{i, j}}\right\rfloor, \quad \forall j,
$$

where, as illustrated and proven by Ye et al. in [12], equal resource allocation among the UE in all cells is optimal for the logarithmic utility; we assume that all the available RBs are allocated in each cell following the round robin algorithm with full bandwidth allocation [13], so that each cell is fully loaded and there will be intercell interference in each RB; $N_{0}$ is the additive white Gaussian noise (AWGN) power; and $I_{i, j}$ is the interference power in a RB received by UE $i$ from BSs other than BS $j$; that is,

$$
I_{i, j}=\sum_{j^{\prime}=1, j^{\prime} \neq j}^{N_{\mathrm{BS}}} P_{j^{\prime}} \cdot g_{i, j^{\prime}}, \quad \forall j \in \mathcal{N}_{\mathrm{BS}} .
$$

The additional small cell deployment optimization problem is formulated as

$$
\begin{aligned}
\underset{\mathbf{U},\left(\mathbf{x}_{1}, \mathbf{y}_{1}\right),\left(\mathbf{x}_{2}, \mathbf{y}_{2}\right)}{\arg \max } & \frac{\sum_{i=1}^{N_{\mathrm{U}}} \gamma_{i}}{N_{\mathrm{U}}} \\
\text { s.t. } & \left(\mathbf{x}_{1}, \mathbf{y}_{1}\right) \in\left|\mathscr{H}_{\mathrm{HS}}\right|, \\
& \left(\mathbf{x}_{2}, \mathbf{y}_{2}\right) \in\left|\mathscr{H}_{\mathrm{NHS}}\right| \\
& \sum_{j=1}^{N_{\mathrm{BS}}} u_{i, j}=1, \quad \forall i \in \mathcal{N}_{\mathrm{U}}, \\
& \gamma_{i} \geq \gamma_{\mathrm{th}}, \quad \forall i \in \mathcal{N}_{\mathrm{U}}, \\
& N_{\mathrm{RB}} \geq N_{j}^{\mathrm{RB}}>0, \quad \forall j \in \mathcal{N}_{\mathrm{BS}}, \\
& u_{i, j} \in\{0,1\}, \quad \forall i \in \mathcal{N}_{\mathrm{U}}, \forall j \in \mathcal{N}_{\mathrm{BS}},
\end{aligned}
$$

where $\mathrm{U}$ is the $N_{\mathrm{U}} \times N_{\mathrm{BS}}$ UE-BS association matrix that contains all $u_{i, j}$ as elements; $\left(\mathbf{x}_{1}, \mathbf{y}_{1}\right)$ are the $N_{\mathrm{NS}_{1}} \times 1$ location vectors of additional small cells in the HS area; $\left(\mathbf{x}_{1}, \mathbf{y}_{2}\right)$ are 
the $N_{\mathrm{NS}_{2}} \times 1$ location vectors of additional small cells in non-HS areas; $\left|\mathscr{H}_{\text {HS }}\right|$ and $\left|\mathscr{H}_{\text {NHS }}\right|$ are the feasible deployment areas [14] for additional small cells in the HS and non-HS areas, respectively; and $\gamma_{\text {th }}$ is the minimum UE throughput threshold.

Note that the joint optimization of the locations of additional small cells and the user associations of all cells in (26) is a mixed integer programming problem, which is NPhard and requires a high computational complexity to find the global optimal solution. Therefore, we propose a practical algorithm in Algorithm 1 to solve the optimization problem in (26).

In Algorithm 1, the binary constraint of user association indicators is relaxed to $0 \leq u_{i, j}^{r} \leq 1$ [15]. All user association indicators are initialized as 0 . Denote $\mathbf{X}_{1}$ and $\mathbf{X}_{2}$ as the sets of additional small cells in the HS and nonHS areas, respectively. The two sets are both initialized as empty. The sizes $N_{\mathrm{NS}_{1}}$ and $N_{\mathrm{NS}_{2}}$ of vectors $\left(\mathbf{x}_{1}, \mathbf{y}_{1}\right)$ and $\left(\mathbf{x}_{2}, \mathbf{y}_{2}\right)$, respectively, are initialized based on the analytical results in Section 3. The optimized numbers of additional small cells to be deployed will be refined in the algorithm. The additional small cells' locations are optimized by the generalized reduced gradient (GRG) method [16].

\section{Simulation Results}

In this section, we present simulation results to evaluate the performance of the proposed algorithm for deploying additional small cells on top of an existing HetNet affected by HS. In the simulation, in addition to Table 2 we set the transmit power of the macro-BS and a small-cell BS as $46 \mathrm{dBm}$ and $23 \mathrm{dBm}$, respectively, the bandwidth of each $\mathrm{RB}$ $W=180 \mathrm{kHz}$, the minimum UE throughput threshold $\gamma_{\mathrm{th}}=$ $3 \mathrm{Mbps}$, the number of RBs in each cell $N_{\mathrm{RB}}=50$, and $N_{0}=-174 \mathrm{dBm} / \mathrm{Hz}$.

Figure 4 shows the average UE throughput with or without additional small cells deployed following our proposed algorithm and with the same number of additional small cells randomly deployed versus the spatial intensity of HS UE. We can see that, by deploying additional small cells following our proposed algorithm, the average UE throughput is much higher than without deploying any additional small cells or randomly deploying the same number of additional small cells. The average UE throughput can be kept above the threshold even for a very high intensity of HS UE. If without deploying additional small cells or randomly deploying the additional small cells the average UE throughput drops below the threshold as the intensity of HS UE increases, indicating that some of the UE will have unsatisfactory QoS.

In order to evaluate the load balancing performance of our algorithm, we calculate the fairness index of all UE [17] as

$$
\mathscr{F}=\frac{\left(\sum_{i=1}^{N_{\mathrm{U}}} \gamma_{i}\right)^{2}}{N_{\mathrm{U}} \cdot \sum_{i=1}^{N_{\mathrm{U}}} \gamma_{i}^{2}} .
$$

The fairness index versus the intensity of HS UE is shown in Figure 5. Since the number and locations of additional small cells and user associations are optimized, the fairness index

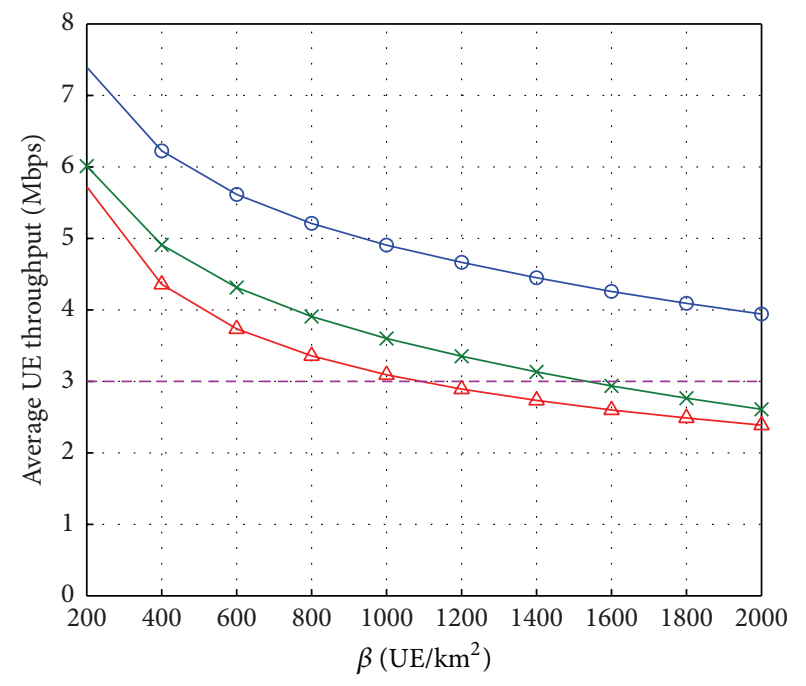

$\_$With additional small cells deployed
$\triangle$ Without additional small cells deployed
$\star \quad$ With additional small cells randomly deployed
--- Minimum UE throughput threshold

FIgURE 4: Average UE throughput versus the intensity of HS UE.

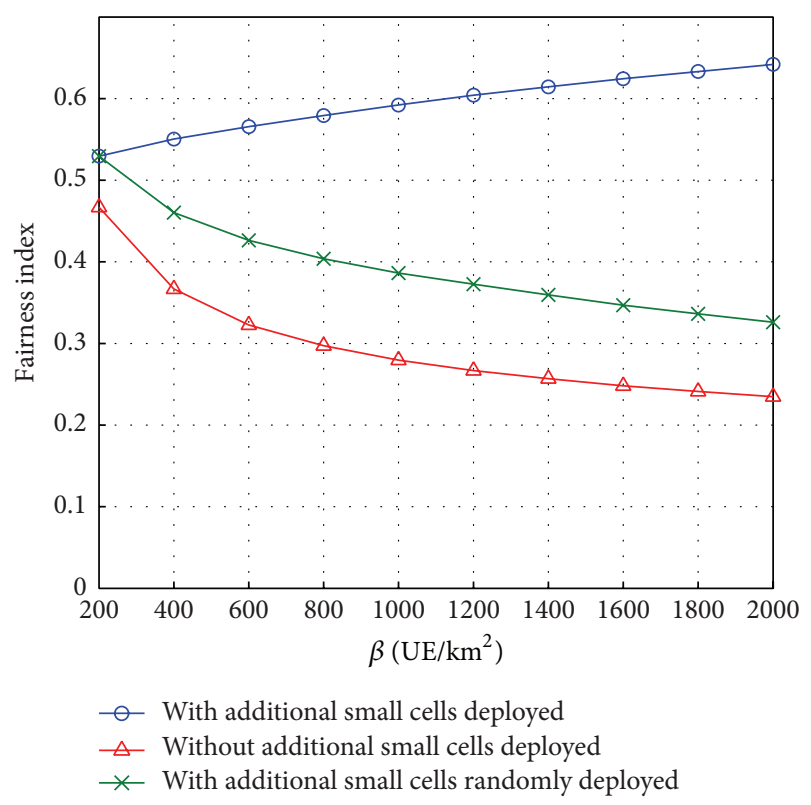

FIGURE 5: Fairness index versus the intensity of HS UE.

achieved by our proposed algorithm is much larger than those without deploying additional small cells or randomly deploying the additional small cells and even increases with the spatial intensity of HS UE. The fairness indexes of those without deploying additional small cells or randomly deploying additional small cells decrease with the spatial intensity of HS UE, indicating that not only the QoS of UE but also the fairness among UE (load balancing performance) is significantly degraded due to the HS. 


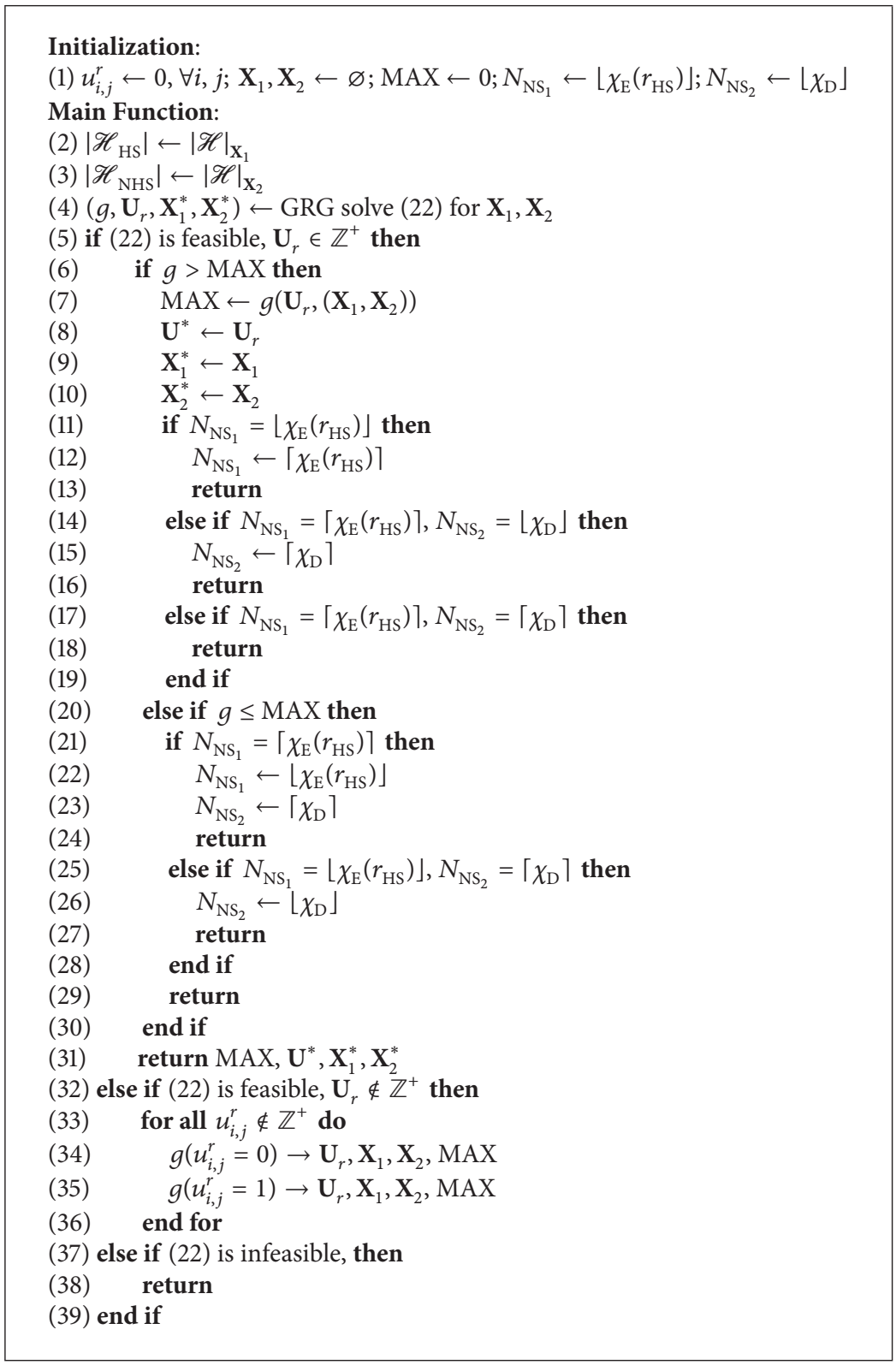

Algorithm 1

Figure 6 shows the area spectral efficiency (ASE) [18] with additional small cells deployed following our proposed algorithm and with the same number of additional small cells randomly deployed versus the spatial intensity of HS UE. We can see that by deploying additional small cells following our proposed algorithm, the ASE is much higher than randomly deploying the same number of additional small cells, and the gain in ASE increases with the intensity of HS UE. This is because the numbers of additional small cells deployed in HS and non-HS areas are, respectively, optimized for given intensity of HS UE, as shown in Figure 3.

We also perform simulation to evaluate the average interference power received by a macro-UE caused by the additional small cells. The simulation result is presented in Figure 7 . We can see that the average macro-UE received interference power caused by additional small cells increases with the number of additional small cells. However, compared with randomly deploying the same number of additional small cells, our proposed algorithm results in much less interference for any given number of additional small cells.

\section{Conclusion}

In this paper, we have analyzed the relationship between the intensities of HS UE (not expected in the original network planning) and additional small cells required to fulfill the extra traffic demand of the HS UE based on a spatial bivariate PPP model of the HetNet. Based on the analytical results, we obtain the optimal numbers of additional small cells for the HS and non-HS areas and propose an algorithm to maximize 


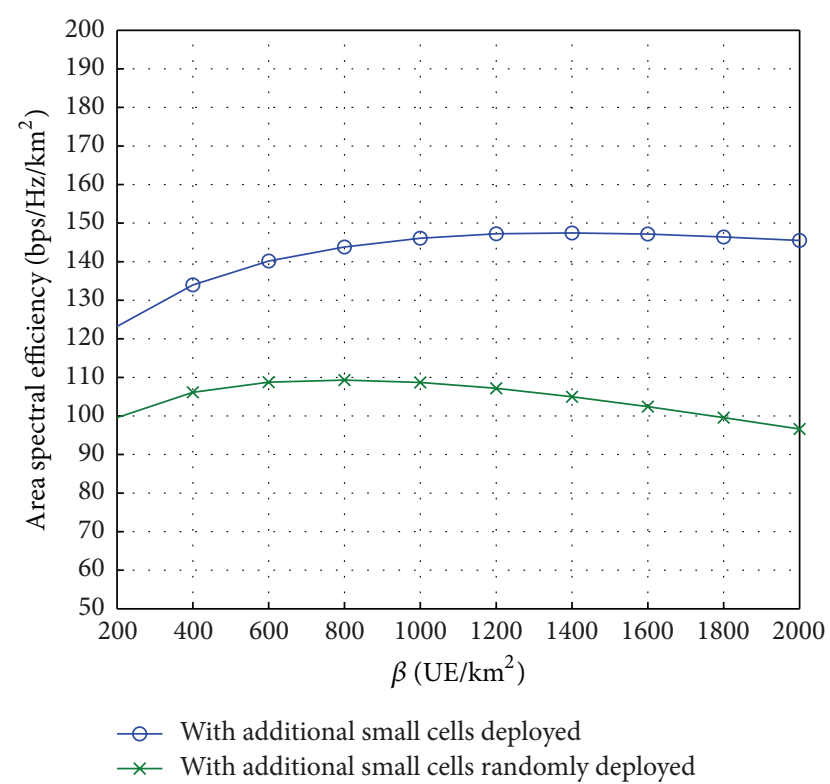

FIGURE 6: Area spectral efficiency versus the intensity of HS UE.

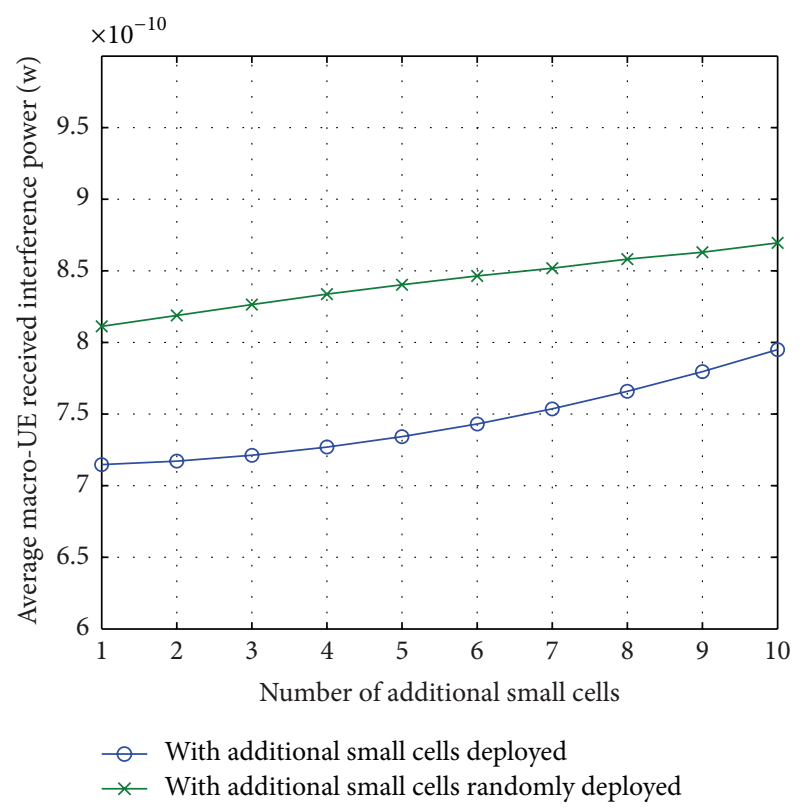

FIGURE 7: Macro-UE received interference power versus the number of additional small cells.

the average UE throughput (after unexpected HS occurs) by jointly optimizing the locations of additional small cells and the user associations of all cells. Simulation results show that the proposed algorithm for optimizing the deployment of additional small cells on top of an existing HetNet can guarantee the QoS requirement of all UE even for a very high density of HS UE while achieving excellent load balancing performance with a lower interference to macro-UE for the HetNet.

\section{Future Work}

In our future work, we plan to extend our work in two directions: (1) stochastic geometry analysis and (2) small cell deployment optimization, both considering the possible occurrence of multiple HSs (with independent HS UE intensities), which is closer to real HetNets. User movement and mobile traffic patterns will also be considered in the small cell deployment algorithm.

\section{Conflict of Interests}

The authors declare that there is no conflict of interests regarding the publication of this paper.

\section{References}

[1] 3GPP TR 36.912 V2.0.0, "3GPP; technical specification group radio access network; feasibility study for further advancements for eutra (release 9)," August 2009.

[2] M. Haenggi, J. G. Andrews, F. Baccelli, O. Dousse, and M. Franceschetti, "Stochastic geometry and random graphs for the analysis and design of wireless networks," IEEE Journal on Selected Areas in Communications, vol. 27, no. 7, pp. 1029-1046, 2009.

[3] A. Babaei and B. Jabbari, "Distance distribution of bivariate Poisson network nodes," IEEE Communications Letters, vol. 14, no. 9, pp. 848-850, 2010.

[4] H.-Y. Hsieh, S.-E. Wei, and C.-P. Chien, "Optimizing small cell deployment in arbitrary wireless networks with minimum service rate constraints," IEEE Transactions on Mobile Computing, vol. 13, no. 8, pp. 1801-1815, 2014.

[5] W. Zhao, S. Wang, C. Wang, and X. Wu, "Cell planning for heterogeneous networks: an approximation algorithm," in Proceedings of the 33rd Annual IEEE International Conference on Computer Communications (INFOCOM '14), pp. 1087-1095, IEEE, Toronto, Canada, April-May 2014.

[6] Y. Park, J. Heo, H. Kim et al., "Effective small cell deployment with interference and traffic consideration," in Proceedings of the 80th IEEE Vehicular Technology Conference (VTC '14), pp. 1-5, IEEE, Vancouver, Canada, September 2014.

[7] S. F. Chou, T. C. Chiu, Y. J. Yu, and A. C. Pang, "Mobile small cell deployment for next generation cellular networks," in Proceedings of the IEEE Global Communications Conference (GLOBECOM '14), pp. 4852-4857, Austin, Tex, USA, December 2014.

[8] M. Tahani and M. Sabaei, "A distributed data-centric storage method for hot spot mitigation in wireless sensor networks," in Proceedings of the International Symposium on Telecommunications (IST '10), pp. 401-408, Tehran, Iran, December 2010.

[9] M. Qutqut, H. Abou-zeid, H. Hassanein, A. Rashwan, and F. Al-Turjman, "Dynamic small cell placement strategies for LTE heterogeneous networks," in Proceedings of the IEEE Symposium on Computers and Communication, pp. 1-6, Madeira, Portugal, June 2014.

[10] T. C. Brown, B. W. Silverman, and R. K. Milne, "A class of twotype point processes," Zeitschrift für Wahrscheinlichkeitstheorie und Verwandte Gebiete, vol. 58, no. 3, pp. 299-308, 1981.

[11] J. E. Paloheimo, "A spatial bivariate poisson distribution," Biometrika, vol. 59, no. 2, pp. 489-492, 1972.

[12] Q. Ye, B. Rong, Y. Chen, M. Al-Shalash, C. Caramanis, and J. G. Andrews, "User association for load balancing in heterogeneous 
cellular networks," IEEE Transactions on Wireless Communications, vol. 12, no. 6, pp. 2706-2716, 2013.

[13] 3GPP TR 36.814 V9.0.0, "Evolved Universal Terrestrial Radio Access (E-UTRA); Further Advancements for EUTRA Physical Layer Aspects (Release 9)," March 2010.

[14] J. Wu, X. Chu, D. López-Pérez, and H. Wang, "Femtocell exclusion regions in hierarchical 3-sector macrocells for cochannel deployments," in Proceedings of the 1st IEEE International Conference on Communications in China (ICCC '12), pp. 541-545, Beijing, China, August 2012.

[15] J. M. Ortega and W. C. Rheinboldt, Iterative Solution of Nonlinear Equations in Several Variables, vol. 30, SIAM, Philadelphia, $\mathrm{Pa}, \mathrm{USA}, 1970$.

[16] L. S. Lasdon, A. D. Waren, A. Jain, and M. Ratner, "Design and testing of a generalized reduced gradient code for nonlinear programming," ACM Transactions on Mathematical Software, vol. 4, no. 1, pp. 34-50, 1978.

[17] R. Jain, D. Chiu, and W. Hawe, A Quantitative Measure of Fairness and Discrimination for Resource Allocation in Shared Computer Systems, vol. 38, Eastern Research Laboratory, Digital Equipment Corporation, Hudson, Mass, USA, 1984.

[18] M.-S. Alouini and A. J. Goldsmith, "Area spectral efficiency of cellular mobile radio systems," IEEE Transactions on Vehicular Technology, vol. 48, no. 4, pp. 1047-1066, 1999. 

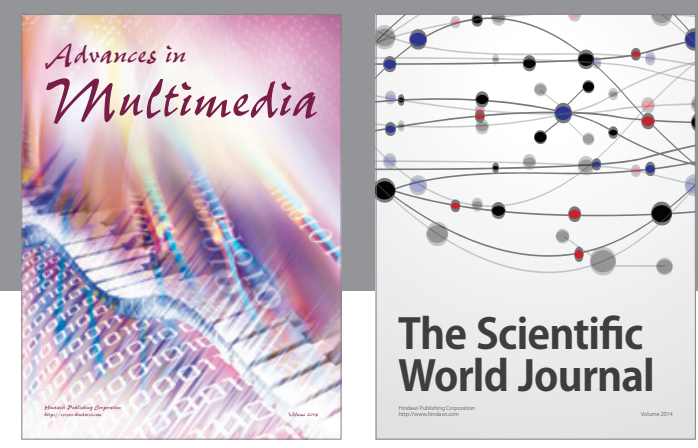

The Scientific World Journal
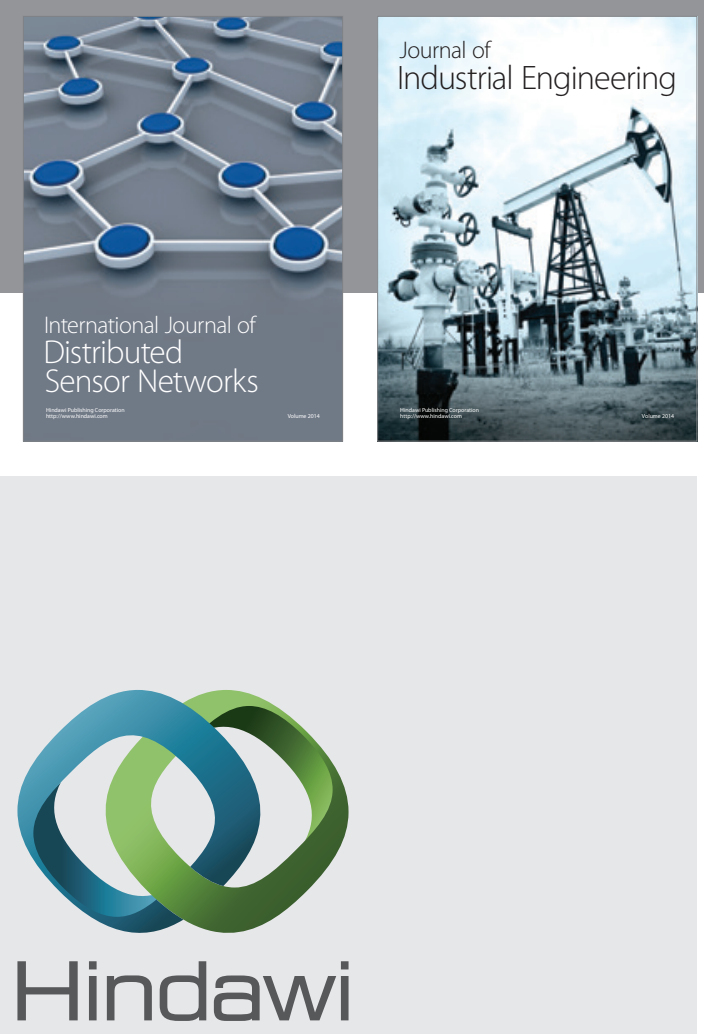

Submit your manuscripts at

http://www.hindawi.com

\section{Computer Networks} and Communications
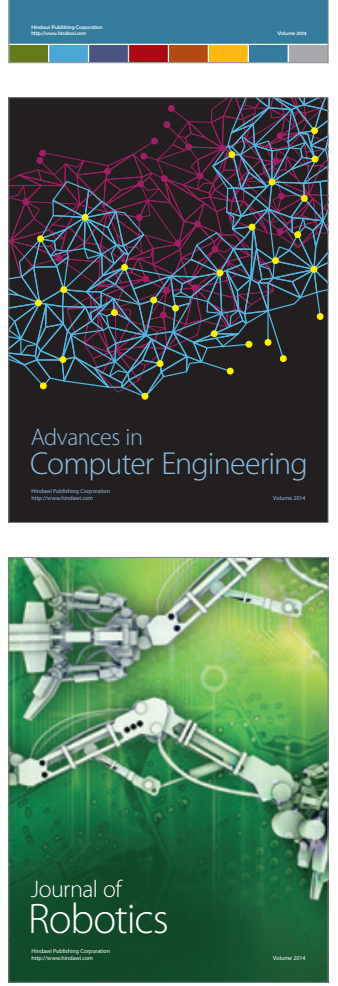
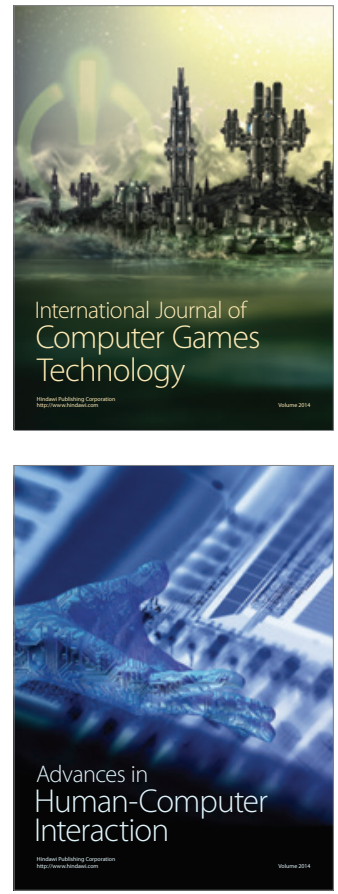
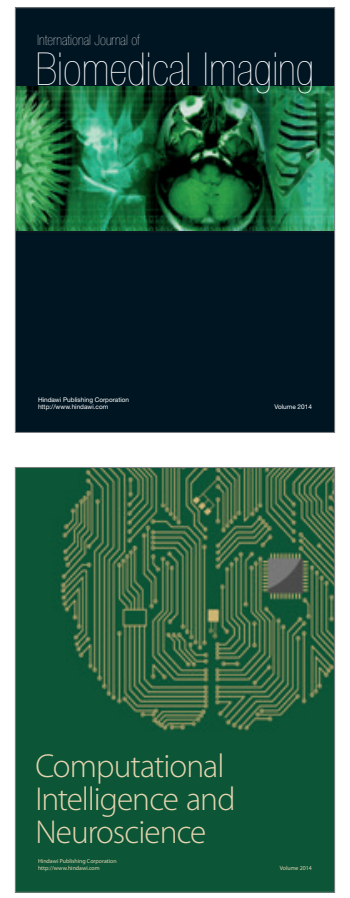
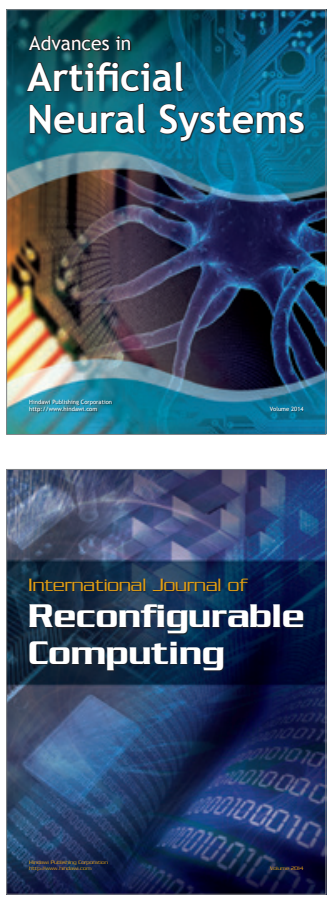
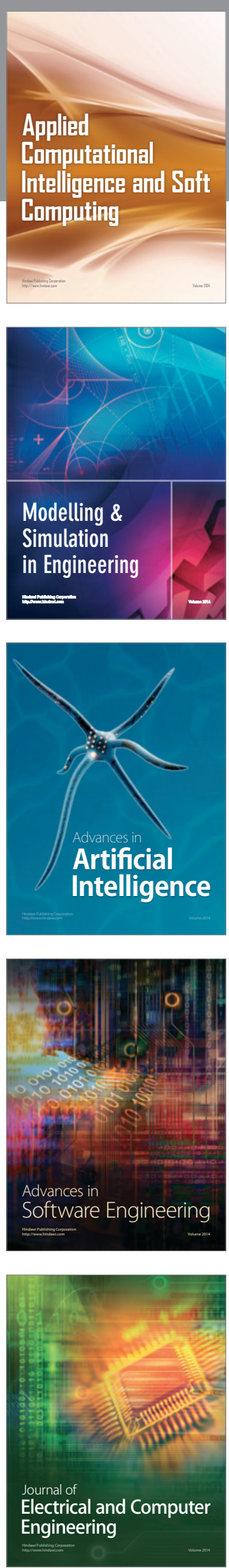